\title{
Peak Detection based Spread Spectrum Audio Watermarking using Discrete Wavelet Transform
}

\author{
Neha Baranwal \\ School of Computer Engineering \\ KIIT, Bhubaneswar
}

\author{
Kamalika Datta \\ School of Computer Engineering \\ KIIT, Bhubaneswar
}

\begin{abstract}
This paper proposes a robust spread spectrum based audio watermarking scheme using Discrete wavelet transform (DWT). Here we use a peak detection algorithm to obtain high robustness. In this watermarking scheme, watermarks are embedded into the peak value of detail coefficients of, transformed audio signal. It is a blind watermarking technique which is used for providing copyright protection and content authentication for digital content. Tests shows that the proposed watermarking scheme is robust against signal processing attacks like resampling, requantization, MP3 compression, noise addition. Performance of this technique is analyzed by calculating the percentage match and SNR values.
\end{abstract}

\section{Keywords}

Audio watermarking, Spread spectrum, Discrete Wavelet Transform, peak detection.

\section{INTRODUCTION}

As the use of digital data like audio, vedio and images had increased, illegal copying and distribution of these digital data had also increased. To overcome these problems, people have come up with many techniques like hardware based copy protection system etc. As these traditional methods do not serve the purpose, therefore some new techniques have been developed. Digital watermarking [1] [2] is one of the very important method, that have been widely used to prevent from illegal copying and distribution.

Audio watermarking [3] [4] is the process in which information is embedded into the audio signal. In other words digital audio watermarking involves the hiding of data with in a digital audio file, so that later it can be used to trace the authenticity of audio file. Audio watermarking techniques satisfy three characteristics which are inaudibility, robustness and bit rate. These Requirements are represented by the magic triangle [5] shown in figure 1. It is called magic triangle because inaudibility is the main requirement of audio watermarking process. Therefore it is presented in the upper portion of the triangle but the other two requirements cannot achieve together which means if data rate is high robustness is low and robustness is high data rate is low. Therefore it is presented in two corners of the triangle.

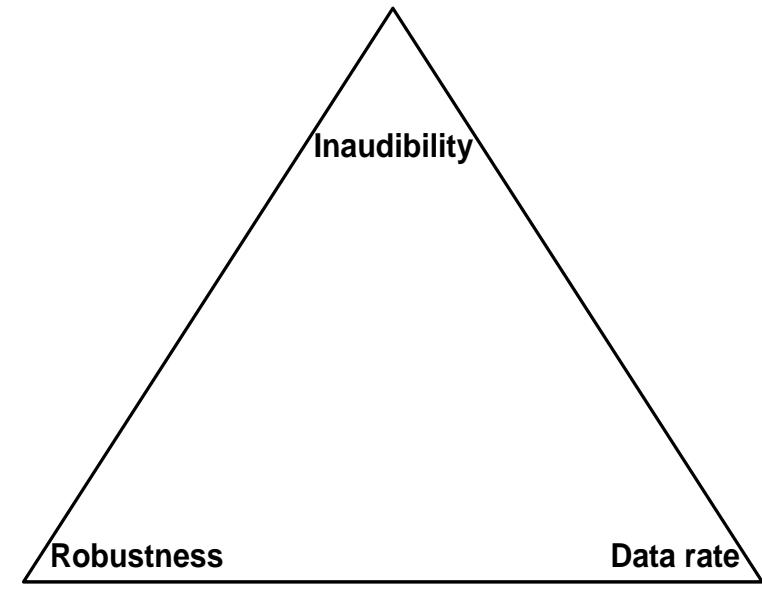

Fig 1: Magic tringle

There are several application areas [2] where digital watermarking technique is used such as copyright protection, copy control, content authentication, fingerprinting, broadcast monitoring etc. In copyright protection copyright owner wants to check the illegal copies of the data. Copy control, this application prevents illegal copying of copyrighted content. Data authentication, in which watermarking is used to check the authentication of data etc. In last few years, various audio watermarking techniques have been proposed [4] [6] such as low-bit coding, phase coding, echo coding and spread spectrum. Among all these techniques, spread spectrum based watermarking techniques are most widely used because this technique provides high robustness against signal processing attacks. In spread spectrum technique lower number of information bits are spreaded into the higher number. Due to spreading nature maximum numbers of information bits are retrieved correctly.

\section{EXISTING WORK}

There exists many SS audio watermarking techniques that have already been developed. Here we will discuss few of the techniques with more focus on SS based watermarking technique. Malvar et al [7] proposed two techniques one is general spread spectrum audio watermarking technique and second one is improved spread spectrum audio watermarking technique. In general audio watermarking scheme information bits are embedded as:

$$
\mathrm{Y}=\mathrm{x}+\alpha \mathrm{w}
$$


where $\mathrm{Y}$ is the watermarked signal, $\mathrm{x}$ is the original signal, $\alpha$ is embedding intensity and $\mathrm{w}$ is the watermark. Here watermark bits are extracted by using normalized correlation mechanism. But this technique fails to provide high robustness. Therefore improved watermarking technique has been developed. This scheme is similar to general spread spectrum audio watermarking scheme, only difference is that linear function $\mu$ $\left(c_{x}, b\right)$ is used in place of $\alpha$ to improved the robustness. In [8] Xing $\mathrm{He}$ et al proposed an audio watermarking scheme using psychoacoustic model. Psychoacoustic model is used for calculating masking threshold value which means upto what level the watermark bits are embedded into audio signal so that it is inaudible to the human ear. In this technique the watermark bits are embedded using discrete wavelet packet transform. Extraction process is the reverse of embedding process. Jung et al [9] proposed an audio watermarking scheme using the spectral envelope filter. This filter is used as a preprocess to enhances the watermark detection performance. In this scheme watermark bits are embedded using MPEG-2 advance audio coding encoding system. In this system quantization and huffman coding is used for embedding of watermark data. In [10] Mingjie Chen et al proposed a frequency selective spread spectrum audio watermarking technique using communication channel model and attack characterization property. Here attack characteristics property is used against signal processing attacks. Here embedding and extraction process is performed by using communication channel model. Dhar et al [11] proposed a frame based audio watermarking scheme using discrete fourier transform(DFT). In this watermarking scheme watermark bits are embedded into the most prominent peak of each frame, so that finding the exact position of these peak values are very difficult. This technique does not provide good robustness against MP3 compression attack. In [12] author proposed a new audio watermarking algorithm using DWT. Here binary image is taken as a watermark. This technique, watermark bits are embedded into the strongest detail coefficients of transformed audio signal. Embedding is performed as:

$$
\mathrm{v}^{\prime}=\mathrm{v}+\alpha \mathrm{x}
$$

where $\mathrm{v}^{\prime}$ is the coefficients after embedding process, $\mathrm{v}$ is the strongest coefficient, $\mathrm{x}$ is the watermark bits. Here the no. of detail coefficients are same as the watermark bits.

We have proposed a spread spectrum based audio watermarking scheme which is used for providing copyright protection of digital audio data. In this technique watermark bits are embedded into the peak values of transformed audio signal. Experimental results demonstrate that the proposed watermarking scheme provides better performance against signal processing attacks such as re-sampling, requantization, MP3 compression etc in comparison to other techniques.

\section{PROPOSED WORK}

In this paper an audio watermarking scheme using spread spectrum technique in DWT domain is proposed. Here the audio signal is first decomposed upto the third and fourth level of detail coefficients using DWT transformation. After that watermark is embedded into the highest peak values of detail coefficients. A collection of $0 \mathrm{~s}$ and $1 \mathrm{~s}$ are used as watermark bits. The mechanism we have used for watermark embedding and extraction purpose is explained below.

\section{- Watermark Embedding Process}

The process of proposed watermark embedding scheme is presented in figure 2 .

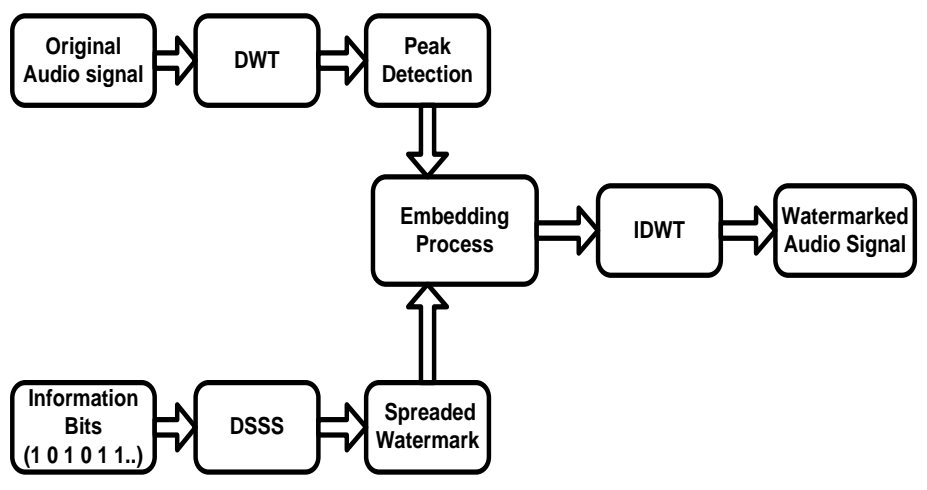

Fig 2. Watermark embedding process

1. The watermark data $w=[1,0,1 \ldots]$ are first spreaded by using the direct sequence spread spectrum technique. In this technique, a pseudo random number is used for spreading the watermark bits. After spreading process the watermark bits are $w^{\prime}(i)=[1,0,1,1 \ldots \ldots . d], 1 \leq i \leq d$, where $\mathrm{d}$ is the length of spreaded watermark which are 100 times greater then the original one.

2. The original audio signal is first sampled into $\mathrm{S}(\mathrm{i}), 1 \leq i \leq$ $N$.

3. Decompose these sample values upto $\mathrm{K}$ levels using discrete wavelet transform. After decomposition, approximate and detail coefficients are $x a_{k}, x d_{k}, x d_{k-l}, x d_{k-}$ ${ }_{2} \ldots \ldots \ldots \ldots \ldots d_{1}$.

4. Calculate the $\mathrm{d}$ highest peak values from the detail coefficient $x d_{k}$ of audio signal and stored in vector $\mathrm{P}$. Indices of the peak values are stored in vector $\mathrm{E}$, where peak values are calculated using Carlos Adrian Vargas Aguilera's extrema function.

5. After that information bits are embedded into these peak values by using the formula:

$$
x d_{K}^{\prime}(E(t))=x d_{K}(E(t))+\alpha * w
$$

where $E(t)$ is the index of $\mathrm{t}^{\text {th }}$ peak value of detail coefficients $(1 \leq \mathrm{t} \leq \mathrm{d})$ and $\alpha$ is a parameter called the embedding intensity.

6. Finally, watermarked signal is obtained by applying IDWT.

After embedding process, watermark bits are extracted as follows:

\section{- Watermark Extraction Process}

The extraction process is presented in figure 3 . 


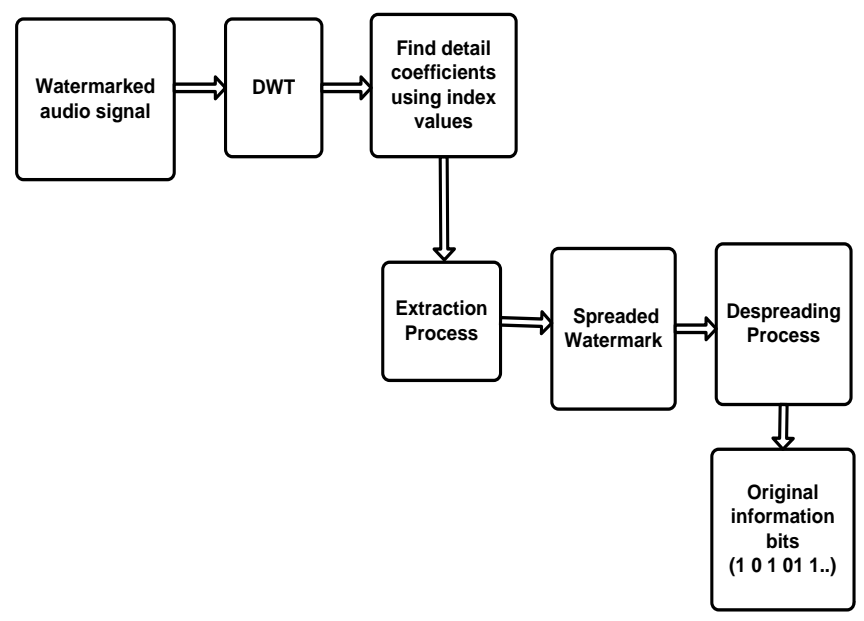

Fig 3. Watermark extraction process

1. Watermarked signal is decomposed upto $K^{\text {th }}$ level using DWT.

2. After decomposition the detail coefficients $\mathrm{xd}_{\mathrm{k}}$ of watermarked audio signal are obtained.

3. Searched those detail coefficients which are present at indices $\mathrm{E}$ are denoted as $P \square$.

4. After that information bits are extracted as follows:

$$
\begin{gathered}
w a=\left(P^{\prime}-P\right) / \alpha \\
w a^{\prime}(i)=\left\{\begin{array}{l}
1 \text { if } w a>1 \\
0 \text { if } w a \leq 1
\end{array}\right.
\end{gathered}
$$

Where, wa' is the spreaded watermark.

5. The original watermarks $w$ are obtained after dispreading of $w a$ '.

\section{SIMULATION RESULTS AND ANALYSIS}

In this section the simulation results of watermarking scheme are presented. The performances of the scheme are analyzed against signal processing attacks such as MP3 compression, resampling, requantization, noise addition. Here the number of watermark bits after spreading process is 1000 . A set of wave files are used such as pop, rock, country, speech etc. We have performed simulations using MATLAB 7.9. In our proposed watermarking scheme Daubechies-2 wavelet transform are used for decomposition of audio files.

- MP3 compression: The robustness of proposed watermarking scheme are tested against MP3 compression attack. In MP3 compression technique, audio files are compressed into very small size so that the audio files are easy to handle. Here the audio files are compressed at 64 Kbps.
- Resampling Attack: In re-sampling attack the watermarked audio signal is re-sampled at a sampling rate of $F s / 2$, where Fs is the original sampling frequency. After that it is recovered back to original frequency Fs.

- Requantization Attack: Here the watermarked audio signal is quantized into 8-bit quantization level.

\section{A. Experiment 1}

In experiment 1, the watermark bits are embedded into the peak values of third level detail coefficients using DWT. Thesimulation results for experiment 1 against signal processing attacks like MP3 compression, resampling and requantization are presented in table 1,2 .

Table 1. MP3 compression attack for 1000 bits

\begin{tabular}{|c|c|c|}
\hline Wav Files & \% match & SNR \\
\hline c1 & 98 & 16.73 \\
\hline c2 & 100 & 15.93 \\
\hline hindi1 & 100 & 21.84 \\
\hline hindi2 & 100 & 22.76 \\
\hline popb1 & 99 & 22.16 \\
\hline popb2 & 96 & 18.25 \\
\hline sam1 & 100 & 16.82 \\
\hline sam2 & 100 & 20.47 \\
\hline
\end{tabular}

\begin{tabular}{|c|c|c|c|}
\hline Wav Files & \% match & $\begin{array}{c}\text { SNR after } \\
\text { resampling }\end{array}$ & $\begin{array}{c}\text { SNR after } \\
\text { re-quant }\end{array}$ \\
\hline c1 & 100 & 19.96 & 26.40 \\
\hline c2 & 100 & 17.73 & 17.45 \\
\hline hindi1 & 100 & 22.07 & 21.60 \\
\hline hindi2 & 100 & 23.37 & 22.90 \\
\hline popb1 & 100 & 25.20 & 28.33 \\
\hline popb2 & 99 & 23.79 & 27.44 \\
\hline sam1 & 100 & 16.99 & 16.31 \\
\hline sam2 & 100 & 20.76 & 20.23 \\
\hline
\end{tabular}

Table 2. Resamp. \& requant. attack for 1000 bits

\section{B. Experiment 2}

Here the watermark bits are embedded into the peak values of fourth level detail coefficients of audio signal obtained using DWT transform. Simulation results of experiment 2 are presented in table 3,4 . 
Table 3. MP3 compression attack for 1000 bits

\begin{tabular}{|c|c|c|}
\hline Wav Files & \% match & SNR \\
\hline $\mathrm{c} 1$ & 97 & 16.73 \\
\hline $\mathrm{c} 2$ & 100 & 15.81 \\
\hline hindi1 & 100 & 21.73 \\
\hline hindi2 & 100 & 22.75 \\
\hline popb1 & 99 & 22.16 \\
\hline popb2 & 97 & 18.33 \\
\hline sam1 & 100 & 16.71 \\
\hline sam2 & 100 & 20.31 \\
\hline
\end{tabular}

Table 4. Resamp. \& requant. attack for 1000 bits

\begin{tabular}{|c|c|c|c|}
\hline Wav Files & \% match & $\begin{array}{c}\text { SNR after } \\
\text { resampling }\end{array}$ & $\begin{array}{c}\text { SNR after } \\
\text { re-quant }\end{array}$ \\
\hline c1 & 100 & 19.89 & 26.61 \\
\hline c2 & 100 & 17.47 & 17.66 \\
\hline hindi1 & 100 & 21.75 & 21.82 \\
\hline hindi2 & 100 & 23.05 & 23.12 \\
\hline popb1 & 100 & 25.05 & 28.82 \\
\hline popb2 & 100 & 23.66 & 18.73 \\
\hline sam1 & 100 & 16.99 & 16.52 \\
\hline sam2 & 100 & 20.76 & 24.30 \\
\hline
\end{tabular}

\section{Experiment 3}

Simulation results of existing scheme [12] are presented in table 5, 6 against different signal processing attacks. Here the watermark bits are embedded into the $\mathrm{N}$ strongest detail coefficients of transformed audio signal.
Table 5. MP3 compression attack for 1000 bits

\begin{tabular}{|c|c|c|}
\hline Wav Files & \% match & SNR \\
\hline c1 & 97 & 16.70 \\
\hline c2 & 100 & 15.74 \\
\hline hindi1 & 100 & 21.54 \\
\hline hindi2 & 100 & 22.51 \\
\hline popb1 & 97 & 22.21 \\
\hline popb2 & 96 & 18.24 \\
\hline sam1 & 100 & 16.51 \\
\hline sam2 & 100 & 20.16 \\
\hline
\end{tabular}

Table 6. Resamp. \& requant. attack for 1000 bits

\begin{tabular}{|c|c|c|c|}
\hline Wav Files & \% match & $\begin{array}{c}\text { SNR after } \\
\text { resampling }\end{array}$ & $\begin{array}{c}\text { SNR after } \\
\text { re-quant }\end{array}$ \\
\hline c1 & 100 & 19.94 & 26.31 \\
\hline c2 & 100 & 19.25 & 17.36 \\
\hline hindi1 & 100 & 21.97 & 21.52 \\
\hline hindi2 & 100 & 23.31 & 22.82 \\
\hline popb1 & 100 & 25.20 & 28.25 \\
\hline popb2 & 100 & 23.75 & 27.36 \\
\hline sam1 & 100 & 16.82 & 16.24 \\
\hline sam2 & 100 & 20.71 & 20.15 \\
\hline
\end{tabular}

From above tables we have seen that the performance of our proposed technique is better than the existing one. Here the proposed scheme provides high percentage match and high SNR values for all the wave files in comparison to existing technique.

\section{CONCLUSION}

A secured audio watermarking based on spread spectrum technique is presented in this paper. A peak detection method is used for embedding of watermark bits. A set of experiments have been performed and their performances are evaluated on the basis of SNR and percentage match. Here the performance of existing method is compared with our proposed one.

In our proposed scheme the watermark bits are extracted without using the original signal. From experimental results we observe that our proposed technique provides low bit error rate and high SNR in comparison to existing techniques. 


\section{REFERENCES}

[1] C. I. Podilchuk and E. J. Delp, "Digital watermarking: Algorithms and applications," IEEE Signal Processing Magazine, vol. 18, pp. 33-46, July 2001.

[2] I. J. Cox, Digital watermarking and stegnography. Morgan kaufmann, 2008.

[3] H. Y. Z. Y. Xianghong Tang, Yamei Niu, "A digital audio watermark embedding algorithm," International Journal of Information Technology, vol. 11, pp. 24-31, 2005.

[4] Chun-ShienLu, Multimedia security: stegnography and digital watermarking techniques for protection of intellectual property. Idea Group publishing, 2004.

[5] N. Cvejic, "Algorithms for audio watermarking and steganography," Master's thesis, Department of Electrical and Information Engineering, Information Processing Laboratory, University of Oulu, 2004.

[6] M. Hrnr and J. Krajovi, "Principles of audio watermarking," Advances in Electrical and Electronic Engineering, pp. 247-249, august 2006.

[7] H. S. Malvar and D. A. F. Florncio, "Improved spread spectrum: A new modulation technique for robust watermarking," vol. 51, pp. 898-905, April 2003.

[8] W. R. Ding and X. Da-wen, "Audio watermarking algorithm based on wavelet packet and psychoacoustic model," in Proceedings of Sixth International Conference on Parallel and Distributed Computing Applications and Technologies (PDCAT'05), December 2008, pp. 812-814.

[9] S. Jung, J. Seok, and J. Hong, "An improved detection technique for spread spectrum audio watermarking with a spectral envelope filter," ETRI Journal, vol. 25, pp. 52-54, 2003.

[10] M. Chen, K. Fan, W. Mo, and S. Zhang, "Implementation and evaluation of a novel audio watermarking scheme," in Proceedings of International Conference on Computational Intelligence and Security, january 2010, pp. 269-272.

[11] P. K. Dhar and M. I. Khan, "A new audio watermarking system using discrete fourier transform for copyright protection," International Journal of Computer Science and Network Security, vol. 10, pp. 35-40, june 2010.

[12] S. A. B. Charmchamras, S. Kaengin and M. Sangworasil, "Audio watermarking technique using binary image in wavelet domain," in Proceedings of International Conference on Information and Communication Systems, December 2007, pp. 1-3.

[13] T. Dutoit and F. Marques, Applied signal processing. Springer Science, 2009.

[14] M. Li, Y. Lei, J. Liu, and Y. Yan, “A novel audio watermarking in wavelet domain," pp. 27-32, December 2006.

[15] M. Adya, "Audio watermark resistant to mp3 compression," Master's thesis, Department Of Computer Science And Engineering Indian Institute Of Technology Kharagpur, India, 2007.

[16] C. Wu, P. Su, and C. J. Kuo, "Robust audio watermarking for copyright protection," Master's thesis, Department of electrical and computer engineering and University of Auckland, 2007. 